\title{
Índices de Utilidad Económica de Corzuela Parda (Mazama gouazoubira)
}

\author{
Utility Indices for Brown Brocket Deer (Mazama gouazoubira) \\ Leonardo Mucciolo y Alejandro Acosta ${ }^{\mathrm{i}}$
}

\section{RESUMEN}

Se presentan resultados de una experiencia de procesamiento de un individuo de corzuela parda (Mazama gouazoubira), a partir de la cual se calculó la utilidad económica de esta especie por medio de los índices MUI (Meat Utility Index), GUI (General Utility Index), y FUI (Food Utility Index). Los resultados se comparan con los de otros cérvidos de mayor tamaño: caribú, huemul, y venado de cola blanca, indicando una correlación altamente significativa y positiva.

Palabras Clave: Índices De Utilidad Económica, Corzuela parda, Zooarqueología

\begin{abstract}
We present results of a butchery experiment of a brown brocket deer (Mazama gouazoubira) individual, which was employed to calculate several utility indexes for this species: MUI (Meat Utility Index), GUI (General Utility Index), y FUI (Food Utility Index). Results are compared with those of larger cervids like caribou, huemul and white-tailed deer, indicating a strong and positive correlation.
\end{abstract}

Keywords: Economic Utility Indices, Brown Brocket deer, Zooarchaeology

CONICET-Instituto Nacional de Antropología y Pensamiento Latinoamericano. 3 de febrero 1378 (C1426BJN).Ciudad Autónoma de Buenos Aires, Argentina. Correo-e: leonardomucciolo@yahoo.com.ar; acosta@retina.ar

Recibido: 04-12-2012 Revisado: 10-01-2014 Aceptado:20-20-2014 


\section{INTRODUCCIÓN}

Desde la publicación de los índices de utilidad de oveja y caribú (Binford 1978), los índices de utilidad se han convertido en un marco de referencia insoslayable para la zooarqueología, dado que ofrecen un indicador objetivo con el cual evaluar las decisiones de transporte y consumo. En virtud de ello, en las últimas décadas se han publicado trabajos que informan la utilidad bruta de productos primarios (órganos, carne, médula y grasa ósea) de distintas especies de mamíferos (ver, entre otros Mengoni Goñalons 199I, Belardi y Gómez Otero 1998, Outram y Rowley-Conwy 1998).

El género Mazama es el más diversificado de los cérvidos sudamericanos, con al menos siete especies (Duarte et al. 2008), entre las que se encuentra la corzuela parda (Mazama gouazoubira, Fischer 18/4). Se trata de un cérvido medianopequeño, de entre II y $25 \mathrm{~kg}$, que habita bosques secundarios, claros de bosque y sabanas arboladas, desde el sur de la floresta amazónica hasta el sur del Chaco sudamericano y Uruguay (Black-Décima et al 2010). Es la especie de Mazama con distribución más extensa en Argentina, con presencia en los sectores boscosos de las provincias fitogeográficas Chaqueña, del Espinal, Paranaense y la base de las Yungas.

En Sudamérica, la corzuela parda y otras especies de Mazama constituyen parte de la dietade diversascomunidades aborígenes, como los Aché (Hill y Padwe 2000) y los Xavante (Coimbra et al 2002). En el Chaco argentino fue explotada por los abipones (Dobrizhoffer 1967/68), y los mocovíes, quienes adicionalmente empleaban su cuero para fabricar cuerdas de arco y parches de tambor, entre otros implementos (Paucke 1944:367).

En términos arqueológicos, la corzuela parda fue tempranamente aprovechada, habiendo sido identificada en niveles datados circa 8000 años $C^{14}$ AP (Nogueira De Queiroz y De Carvalho 2008). En Argentina se han recuperado restos en sitios localizados en el Chaco meridional (Kraglievich y Rusconi 1931) y en el humedal del Paraná inferior (Cione et al 1977; Cione y Tonni 1978). También se han registrado especímenes de Mazama sp. en sitios localizados dentro de su área de distribución (ver del Papa et al 2012, Medina y Pastor 2012, Santini y de Santis 2012). Con respecto a esto último debe destacarse que los rangos de movilidad propios de las sociedades aborígenes establecen la posibilidad de que en los registros exista superposición entre corzuela parda y cérvidos de tamaño similar, tales como Mazama americana, Mazama nemorivaga, Mazama bororo, Mazama nana y Ozotoceros bezoarticus, dificultando su identificación y la evaluación de su importancia económica (ver Acosta et al. 20l I).

En este trabajo, se presentan los Índices de Rendimiento Económico obtenidos a partir del procesamiento de un individuo de corzuela parda. Los índices de utilidad constituyen un importante marco de referencia para evaluar el potencial transporte $y$ procesamiento diferencial de las presas en función de la disponibilidad de productos primarios que brindan las unidades anatómicas (cf.Binford 1978). En el caso de los cérvidos, éstos han sido calculados, con variantes metodológicas, para caríbú (Rangifer tarandus), huemul (Hippocamelus bisulcus) y venado de cola blanca (Odocoelius virginianus) (Binford 1978, Metcalfe y Jones 1988, Belardi y Gómez Otero 1998, Madrigal y Holt 2002). Si bien nuestra intención es brindar un nuevo marco de referencia susceptible de ser aplicado en conjuntos de corzuela parda $y / 0$ de especies similares en peso, nos pareció adecuado agregar una comparación con los cérvidos anteriormente mencionados, dado que podría existir variabilidad en la anatomía económica debido a diferencias en el tamaño corporal, hábitat y/o conductas locomotoras (cf. Madrigal y Holt 2002).

\section{MATERIALESY MÉTODOS}

Se utilizó un macho adulto de corzuela parda (CFA- I 2857) depositado en la Fundación de Historia Natural Félix de Azara. El ejemplar, proveniente de la Estación de Cría de Animales Silvestres deVilla Elisa, (Buenos Aires), murió a causa de lesiones ocasionadas por otro individuo y se encontraba en buen estado de salud. Fue transportado el día de su muerte y congelado hasta el día anterior a su procesamiento'.

El procesamiento fuellevado a cabo empleando bisturí y cuchillos. Siguiendo un criterio similar al de Mengoni Goñalons (1991), se definieron seis unidades mayores de trozamiento:i) cabeza: cráneo y mandíbula; ii) pecho: esternón y costillar; iii) columna vertebral: vértebras cervicales, torácicas y lumbares; iv) cintura pélvica: pelvis, sacro y vértebras caudales; v) cuarto delantero: escápula, húmero, radio, cúbito, carpianos, 
metacarpo y falanges; vi) cuarto trasero: fémur, rótula, tibia, tarsianos, metatarso y falanges.

Para la obtención de la carne, cada unidad de trozamiento mayor fue procesada siguiendo los límites de las unidades anatómicas, aunque notamos que el ángulo de corte resultó una variable fundamental en algunas incisiones, en especial en la segmentación del esqueleto apendicular (fémur y escápula). Se intentó recuperar la totalidad del tejido, aunque en el esqueleto axial, en especial en las vértebras, pequeños restos de carne permanecieron adheridos al hueso. Este proceso fue cronometrado con reloj digital.

Para el cálculo de la utilidad, las unidades anatómicas fueron pesadas empleando una balanza digital. Las mismas fueron pesadas con la carne adherida, y luego de procesadas se pesó la carne y los huesos frescos por separado. En el caso de las unidades anatómicas pares, fueron sumados los pesos de ambos lados y luego promediados. Los órganos -excepto el corazón-y las vísceras no fueron cuantificados, aunque se calcularon valores aproximados por diferencia de peso. La pelvis fue pesada con el sacro y las vértebras caudales. Debido al pequeño tamaño del ejemplar, optamos por procesar el atlas y axis junto al resto de las vértebras cervicales. Del mismo modo, la fracción ósea de la mandíbula fue pesada con el cráneo, aunque la carne de ambas unidades fue pesada separadamente. El seso fue recuperado, aunque probablemente hayan quedado restos dentro del cráneo. Las costillas se pesaron con el esternón. En el esqueleto apendicular, el radio fue cuantificado con el cúbito, $y$ las falanges fueron pesadas en conjunto. La médula ósea no pudo ser recuperada debido a que el esqueleto debía permanecer inalterado para su empleo como material comparativo. Se almacenaron $\mathbf{7 0 0}$ grs. de carne para análisis nutricional.

La esqueletización fue completada mediante exposición aeróbica y sin productos químicos. Dos meses después de la experiencia, los elementos fueron pesados nuevamente, repitiendo la operación seis meses más tarde sin observarse variación. Estos resultados fueron utilizados para los valores de huesos "secos".

\section{Índices calculados}

Se calcularon tres índices de utilidad. Para evitar ambigüedades terminológicas, a continuación se definen cada uno.
El Índice de Utilidad de Carne o Índice de Carne (MUl sensu Outram y Rowley-Conwy 1998) mide la utilidad de carne asociada a cada unidad anatómica.A diferencia del índice del mismo nombre propuesto por Binford (1978), que incluye carne, médula y grasa, el MUI de Outram y Rowley-Conwy (1998) es un "verdadero" índice de carne. Fue calculado de la siguiente manera:

$$
\begin{gathered}
\text { MUI = peso total de la unidad } \\
\text { anatómica - peso del hueso fresco }
\end{gathered}
$$

El peso total corresponde al peso de cada unidad antes de ser procesada la carne, $y$ el peso del hueso fresco es el peso de la misma unidad luego de dicha tarea.

El Índice de Utilidad General (GUI sensu Outram y Rowley-Conwy 1998) mide el valor nutritivo total de cada unidad anatómica, y fue calculado a partir de la formula simplificada propuesta por Metcalfe y Jones para el MUI:

$$
\begin{gathered}
\text { GUI = peso total de la unidad } \\
\text { anatómica - peso del hueso seco }
\end{gathered}
$$

Al igual que en la formula anterior,el peso total es el de cada unidad antes de ser extraída la carne, mientras que el peso del hueso seco es el de la misma unidad luego de completado el proceso de esqueletización. De esta manera se obtiene un valor de la utilidad combinada en términos de carne, médula y grasa de cada unidad anatómica (Metcalfe y Jones 1988: 489).

Finalmente, con el objeto de jerarquizar los denominados “acompañantes", el GUI fue empleado para calcular el Food Utility Index (FUI), empleando el método desarrollado por Binford para derivar el MGUI y explicitado por Metcalfe y Jones (1988:503), que consiste en aumentar el valor de cada unidad a partir de la unidad proximal de mayor valor, que son la escápula en el cuarto delantero y el fémur en el cuarto trasero. Este índice fue calculado en dos series, una para huesos completos, y otra para los segmentos proximales y distales de cada unidad anatómica. Todos los índices fueron estandarizados a partir del valor más alto de cada unidad y/o parte.

Por último, a fines de comparar la anatomía económica de la corzuela parda con la de cérvidos de mayor tamaño, se realizaron correlaciones de los índices obtenidos con los valores de utilidad de caribú, huemul y venado de cola blanca. 


\section{RESULTADOS}

El peso total de carne, hueso, cuero y órganos, y el de cada una de las unidades primarias de trozamiento se presentan en las tablas 1 y 2 respectivamente. El individuo no presentaba astas. El peso del individuo vivo fue de $17.800 \mathrm{~g}$. El peso de la carcasa eviscerada fue de $11.209 \mathrm{~g}$, un $63 \%$ del peso vivo. La fracción cárnica fue del $48,58 \%$ del peso total, distribuidaen un porcentaje ligeramente mayor en el esqueleto axial que en el apendicular ( $5.73 \mathrm{I}$ vs $5.478 \mathrm{~g}$ ).

\section{Composición nutricional}

Una muestra de tejido muscular fue procesada en el Departamento de Química Orgánica de la Facultad de Ciencias Exactas y Naturales de la Universidad de Buenos Aires. El valor calórico obtenido fue de $102 \mathrm{kcal} / 100 \mathrm{~g}$, igual al del impala (cf. Ledger 1968 en Egeland y Bierly 2005) y en un orden intermedio entre la carne vacuna $(96 \mathrm{kcal} / \mathrm{I} 00$ g) y la ovina (I $20 \mathrm{kcal} / \mathrm{l} 00 \mathrm{~g}$ ) (Malec com. pers. 20I I). El porcentaje de grasas en la carne es bajo $(1,60 \%)$, aunque con una buena cantidad de proteínas $(21,75 \%)$.

\section{Índice de utilidad de carne (MUI)}

Se observó notable disparidad entre miembros delanteros y traseros, con el fémur como la unidad con mayor proporción cárnica (ver tabla 3). Esto es congruente con la morfología típica de los cérvidos que prefieren hábitats cerrados, que presentan extremidades posteriores bastante más grandes que las anteriores, adaptadas a carreras cortas y el tránsito en bosques densos (Merino y Rossi 2010). No se localizaron depósitos de grasa subcutánea.

\section{Índice de Utilidad General (GUI)}

Las proporciones de utilidad económica no variaron significativamente, aunque en este caso las costillas ocupan el primer lugar en el ranking (tabla 3). Un dato interesante es que, si bien los huesos perdieron $62,60 \%$ de su peso fresco, esta pérdida se localiza principalmente en el esqueleto axial $(67,48 \%$ vs $46,14 \%$ ) debido fundamentalmente a la presencia diferencial de tejido remanente. Esto significa que una proporción desigual de alimento permaneció en cada unidad anatómica luego de procesada su carne: mientras el GUI remanente del esqueleto apendicular se concentra en los autopodios (elementos que, al no tener carne, presentan toda su utilidad en su cavidad interna), todas las unidades del esqueleto axial (en especial el cráneo) disponen de proporciones significativas de masa edible luego de la extracción inicial de la carne. Este dato tiene implicancias para discutir el potencial de transporte de cada unidad anatómica.

\begin{tabular}{|l|c|}
\hline \multicolumn{1}{|c|}{ Mazama gouazoubira } & Peso (g) \\
\hline Total individuo & 17.800 \\
\hline Total carcasa* & 11.209 \\
\hline Total carne & 8.649 \\
\hline Total huesos & 2.425 \\
\hline Lengua & 53,5 \\
\hline Ojos & 32 \\
\hline Corazón & 166,5 \\
\hline Sesos & 81,5 \\
\hline $\begin{array}{l}\text { Otros órganos, vísceras y sangre } \\
\text { (aproximado) }\end{array}$ & $4.445,5$ \\
\hline $\begin{array}{l}\text { Cuero } \\
\text { * Excluye cuero, ojos, sangre, vísceras y } \\
\text { órganos (excepto los sesos) }\end{array}$ & 1.947 \\
\hline
\end{tabular}

Tabla 1: Peso total de carne, huesos, cuero y órganos de corzuela parda

Table 1: Total weight of meat, bones, skin and organs of brown brocket deer

\begin{tabular}{|c|c|}
\hline Mazama gouazoubira & Peso (g) \\
\hline \multicolumn{2}{|c|}{ Unidades primarias de trozamiento } \\
\hline I) Cabeza & 677,5 \\
\hline 2) Pecho & $1.587,5$ \\
\hline 3) Columna vertebral & $2.649,5$ \\
\hline 4) Cintura pélvica & 816,5 \\
\hline 5) Cuarto delantero & 1.769 \\
\hline 6) Cuarto trasero & 3.709 \\
\hline
\end{tabular}

Tabla 2. Peso de las unidades primarias de trozamiento de corzuela parda

Table 2. Weight of primary butchery units of brown brocket deer

Índice de Utilidad Alimenticia (FUI)

Con los valores obtenidos, se realizaron los ajustes para elementos de menor valor del esqueleto apendicular.Teniendo en cuenta que Metcalfe y Jones (1988: tablas 2 y 3 ) incorporaron un valor de FUI 
para las astas (igual a I), y consideraron el cráneo como "acompañante" (ver Metcalfe y Jones 1988: tablas 2 y 3), optamos por seguir el mismo criterio y generar dos valores para el cráneo + mandíbula, uno considerando esta unidad como "acompañante", y otra sin hacerlo. En este sentido, si bien realizamos este ajuste para hacer el índice comparable con los resultados de otros cérvidos, no creemos que el mismo sea necesario en este caso, habida cuenta del pequeño tamaño de las astas de esta especie.

\begin{tabular}{|c|c|c|c|c|c|c|c|c|}
\hline \multicolumn{9}{|c|}{ Mazama gouazoubira } \\
\hline \multirow{3}{*}{ Unidad anatómica } & \multicolumn{2}{|c|}{ Peso (g) } & \multirow[b]{2}{*}{ MUI } & \multirow[b]{2}{*}{ MUI (s) } & \multirow[b]{2}{*}{ GUI } & \multirow[b]{2}{*}{ GUI (s) } & \multirow[b]{2}{*}{ FUI } & \multirow[b]{2}{*}{ FUI (s) } \\
\hline & Total & $\begin{array}{c}\text { Hueso } \\
\text { seco }\end{array}$ & & & & & & \\
\hline & - & - & - & - & - & - & 1 & 1 \\
\hline \multicolumn{9}{|l|}{ Cráneo } \\
\hline con sesos & - & - & 121,5 & 9,2 & - & - & - & - \\
\hline sin sesos & - & - & 40 & 3,03 & - & - & - & - \\
\hline \multicolumn{9}{|l|}{ Mandíbula } \\
\hline \begin{tabular}{|l|} 
con lengua \\
\end{tabular} & - & - & 120,75 & 9,14 & - & - & - & - \\
\hline sin lengua & - & - & 67,25 & 5,09 & - & - & - & - \\
\hline \multicolumn{9}{|l|}{ Cráneo + mandíbula } \\
\hline con lengua' & 570,25 & 140 & - & - & 430,25 & 28,78 & 430,25 & 28,78 \\
\hline con lengua ${ }^{2}$ & - & - & - & - & - & - & 215,63 & 14,42 \\
\hline sin lengua' & 516,75 & 140 & - & - & 376,75 & 25,2 & 376,75 & 25,2 \\
\hline sin lengua ${ }^{2}$ & - & - & - & - & - & - & 188,88 & 12,63 \\
\hline Cervicales & 832 & 61,5 & 644,5 & 48,81 & 770,5 & 51,54 & 770,5 & 51,54 \\
\hline Torácicas & 856 & 63,5 & 600 & 45,44 & 792,5 & 53,01 & 792,5 & 53,01 \\
\hline Lumbares & 961,5 & 51,5 & 796,5 & 60,32 & 910 & 60,87 & 910 & 60,87 \\
\hline Costillas + esternón & $1.587,50$ & 92,5 & 1310,5 & 99,24 & $1.495,00$ & 100 & $1.495,00$ & 100 \\
\hline $\begin{array}{l}\text { Pelvis + sacro + vértebras } \\
\text { caudales }\end{array}$ & 408,25 & 36 & 296,25 & 22,43 & 372,25 & 24,9 & 372,25 & 24,9 \\
\hline Escápula & 370,75 & 17,5 & 331,5 & 25,1 & 353,25 & 23,63 & 353,25 & 23,63 \\
\hline Húmero & 334 & 27,25 & 277 & 20,98 & 306,75 & 20,52 & 330 & 22,07 \\
\hline proximal & - & - & - & - & - & - & 353,25 & 23,63 \\
\hline distal & - & - & - & - & - & - & 330,00 & 22,07 \\
\hline \multirow{3}{*}{$\begin{array}{l}\text { Radio + cúbito } \\
\text { proximal } \\
\text { distal }\end{array}$} & 126,5 & 24 & 87,75 & 6,65 & 102,5 & 6,86 & 216,25 & 14,46 \\
\hline & - & - & - & - & - & - & 216,25 & 14,46 \\
\hline & - & - & - & - & - & - & 159,38 & 10,66 \\
\hline \multirow{3}{*}{$\begin{array}{l}\text { Metacarpo + carpianos } \\
\text { proximal } \\
\text { distal }\end{array}$} & 28 & 18,75 & 0 & 0 & 9,25 & 0,62 & 112,75 & 7,54 \\
\hline & - & - & - & - & - & - & 46,78 & 3,13 \\
\hline & - & - & - & - & - & - & 28,02 & 1,87 \\
\hline \multirow{3}{*}{$\begin{array}{l}\text { Fémur } \\
\text { proximal } \\
\text { distal }\end{array}$} & I.428,75 & 49 & 1320,5 & 100 & $1.379,75$ & 92,29 & I,379,75 & 92,29 \\
\hline & - & - & - & - & - & - & $1.379,75$ & 92,29 \\
\hline & - & - & - & - & - & - & $1.379,75$ & 92,29 \\
\hline \multirow{3}{*}{$\begin{array}{l}\text { Tibia + calcáneo + astrágalo } \\
\text { proximal } \\
\text { distal }\end{array}$} & 351,5 & 63,25 & 248,5 & 18,82 & 288,25 & 19,28 & 834 & 55,79 \\
\hline & - & - & - & - & - & - & 834,00 & 55,79 \\
\hline & - & - & - & - & - & - & 561,13 & 37,53 \\
\hline \multirow{3}{*}{$\begin{array}{l}\text { Metatarso + tarsianos } \\
\text { proximal } \\
\text { distal }\end{array}$} & 44,5 & 27,75 & 0 & 0 & 16,75 & 1,12 & 425,38 & 28,45 \\
\hline & - & - & - & - & - & - & 152,84 & 10,22 \\
\hline & - & - & - & - & - & - & 84,80 & 5,67 \\
\hline \multirow{3}{*}{$\begin{array}{l}\text { Falanges delanteras + traseras } \\
\text { delanteras } \\
\text { traseras }\end{array}$} & 4,58 & 1,48 & 0 & 0 & 3,10 & 0,21 & 29,76 & 1,99 \\
\hline & 4,21 & 1,33 & 0 & 0 & 2,88 & 0,19 & 57,82 & 3,87 \\
\hline & 4,96 & 1,63 & 0 & 0 & 3,33 & 0,22 & 214,35 & 14,34 \\
\hline
\end{tabular}

Tabla 3: Índices de Utilidad de Carne (MUI), Utilidad General (GUI) y Utilidad Alimenticia (FUI) de corzuela parda Table 3: Meat Utility (MUI), General Utility (GUI) and Food Utility (FUI) indices for brown brocket deer 


\begin{tabular}{|c|c|c|c|c|c|c|c|c|c|}
\hline \multirow{3}{*}{ TAXON } & \multirow{3}{*}{$\begin{array}{l}\text { PESO } \\
(\mathrm{kg})\end{array}$} & \multirow{2}{*}{\multicolumn{2}{|c|}{ MUI }} & \multirow{2}{*}{\multicolumn{2}{|c|}{$\begin{array}{c}\text { GUI } \\
\text { Huesos } \\
\text { completos }\end{array}$}} & \multicolumn{4}{|c|}{ FUI } \\
\hline & & & & & & \multicolumn{2}{|c|}{ Porciones } & \multirow[b]{2}{*}{$r$} & \multirow[b]{2}{*}{ NC } \\
\hline & & $r$ & $\mathrm{NC}$ & $r$ & $\mathrm{NC}$ & $\mathrm{R}$ & $\mathrm{NC}$ & & \\
\hline \multirow{2}{*}{$\begin{array}{l}\text { Venado de cola } \\
\text { blanca }\end{array}$} & \multirow{2}{*}{$35-135$} & Serie NJ' $=0,73^{* *}$ & \multirow{3}{*}{10} & & & & & & \\
\hline & & Serie $\mathrm{TN}^{2}=0,83^{*}$ & & & & & & & \\
\hline Huemul $^{3}$ & $55-90$ & $0,91 *$ & & $0,91 *$ & 10 & $0,89 *$ & 10 & & \\
\hline Caribú ${ }^{4}$ & $80-180$ & & & $0,92 *$ & 14 & $0,90 *$ & 14 & $0,94 *$ & 24 \\
\hline \multicolumn{10}{|l|}{$* \mathrm{p}<0,0 \mathrm{I}$} \\
\hline \multicolumn{10}{|c|}{$\begin{array}{l}\text { ' Datos de Madrigal y Holt (2002) } \\
{ }^{2} \text { Datos de Madrigal (2004) } \\
{ }^{3} \text { Datos de Belardi y Gómez Otero (1998) } \\
{ }^{4} \text { Datos de Metcalfe y Jones (1988) }\end{array}$} \\
\hline
\end{tabular}

Tabla 4: Correlaciones entre índices de corzuela parda y venado de cola blanca, huemul y caribú

Table 4: Correlation between brown brocket deer, white-tailed deer, huemul and caribou utility indices

\section{Comparación con resultados de cérvidos de mayor tamaño}

En primer lugar se compararon los resultados obtenidos con los valores de carne de huemul y venado de cola blanca ${ }^{2}$. Los resultados indican una correlación positiva y significativa (ver tabla 4). Luego el GUI de corzuela parda fue comparado con el de huemul y caribú (no se dispone de valores para venado de cola blanca), indicando correlaciones también altas y significativas (ver tabla 4). Por último comparamos valores del FUI de corzuela parda, huemul y caribú (ver tabla 4). Se realizaron dos series, una para huesos completos y otra para segmentos proximales y distales de huesos largos. En esta última serie sólo se correlacionaron los índices de corzuela parda y caribú. Para facilitar la comparabilidad, en las series de corzuela parda se consignaron los valores de cráneo + mandíbula sin lengua, dado que son los utilizados por Metcalfe $y$ Jones (1988: tablas 2 y 3 ).

\section{CONCLUSIÓN}

Los cérvidos de mediano porte, y en particular la corzuela parda, constituyen recursos de importancia para los aborígenes del Neotrópico sudamericano. Este trabajo ha pretendido aportar una herramienta para evaluar las estrategias empleadas para su procesamiento y transporte a partir del registro arqueofaunístico. Los resultados indican una similitud bastante precisa entre la anatomía económica de corzuela parda y la de cérvidos de mayor tamaño y/o adaptados a ambientes más abiertos.

Agradecimientos: Agradecemos a la Fundación de Historia Natural Félix de Azara, especialmente a Sergio Bogan, quien facilitó el acceso al ejemplar y realizó el procesamiento. Esta investigación se desarrolló en el marco de los proyectos PICT-FONCYT 2035-20II y PIPCONICET I I220II0I00565.

\section{NOTAS}

I El descongelado del ejemplar podría haber modificado ligeramente su contenido de agua.

2 No se dispone de valores de utilidad de carne para caribú con la metodología empleada aquí. En el caso del venado de cola blanca se utilizaron dos series, una obtenida por Madrigal y Holt (2002), y otra por Jacobson (2000, en Madrigal y Holt 2002).Ambas series difieren fundamentalmente en el criterio empleado para procesar algunas unidades, puesto que Madrigal y Holt (2002) consideraron los límites "naturales", es decir, la localización de los paquetes musculares, en tanto que la serie de Jacobson (2000) fue realizada considerando los límites exactos de cada hueso (criterio que hemos seguido nosotros). Asimismo, se realizaron algunos ajustes para asegurar la comparabilidad de los resultados: en la serie de huemul de Belardi y Gómez Otero (1998), las costillas y el esternón fueron cuantificadas separadamente y nosotros optamos por sumar ambas unidades. Asimismo, la carne de cráneo y mandíbula no fue considerada puesto que ninguno 
de los índices utilizados para comparar dispone de datos de esas unidades.

\section{BIBLIOGRAFÍA}

Acosta, A., Mucciolo, L., Musali, J., y M. P. Arrizurieta. 2011. "Avances y problemas relacionados con el estudio del registro arqueofaunístico generado por los grupos cazadores-recolectores del extremo sur de la provincia de Entre Ríos (humedal del río Paraná inferior)". En Avances y perspectivas en la Arqueología del Nordeste, editado por M. R. Feuillet Terzaghi, M. B. Colasurdo, J. L. Sartori y S. Escudero, Pp. 43-58. ST Servicios Gráficos, Buenos Aires.

Belardi, J. B. y J. Gómez Otero. 1998. "Anatomía económica del huemul (Hippocamelus bisulcus): una contribución a la interpretación de las evidencias arqueológicas de su aprovechamiento en Patagonia". Anales del Instituto de la Patagonia 26: 195-207

Binford, L. R. 1978. Nunamiut ethnoarchaeology.Academic Press, New York.

Black-Décima, P., R. V. Rossi, A. Vogliotti, J. L. Cartes, L. Maffei, J. M. B. Duarte, S. González y J. P. Juliá. 2010. "Brown brocket deer Mazama gouazoubira (Fischer I8I4)". En Neotropical Cervidology. Biology and medicine of Latin American deer, editado por J. M. B. Duarte y S. González, pp. 19020I. FUNEP/IUCN, Brasil.

Cione, A L., Rizzo, A. y E. P. Tonni. 1977. "Relación cultura aborigen-ambiente en un sitio de Rincón de Landa, Gualeguaychú, Entre Ríos, República Argentina. Nota preliminar". V Encuentro de Arqueología del Litoral, pp. I23-I4I.

Cione, A. y E. P. Tonni. 1978. "Paleoethnozoological context of a site of Las Lechiguanas islands, Parana Delta, Argentina". El Dorado. A Newsletter Bulletin on South American Anthropology III (I): 76-86.

Coimbra, C. E. A., Flowers, N. M., Salzano, F. M. y R. V. Santos. 2002. The Xavánte in Transition: Health, Ecology and Bioanthropology in Central Brazil. Linking Levels of Analysis Series. The University of Michigan Press. Ann Arbor, Michigan.

Del Papa, L. M., De Santis, L., y J. L. Togo. 2012. "Zooarqueología santiagueña. Despertando de la siesta". En Temas de Arqueología 3: Estudios tafonómicos y zooarqueológicos (II), compilado por A. Acosta, Loponte, D. y L. Mucciolo, Pp. I-24. Instituto Nacional de Antropología y Pensamiento Latinoamericano. Buenos Aires.

Dobrizhoffer, M. 1967/68. Historia de los Abipones. Universidad Nacional del Nordeste, Facultad de Humanidades, Departamento de Historia. Resistencia.

Duarte, J. M. B., S. González y J.E. Maldonado. 2008. "The surprising evolutionary history of South American deer”. Molecular Phylogenetics and Evolution 49: 17-22.
Egeland, C. P. y R. M. Byerly. 2005. "Application of Return Rates to Large Mammal Butchery and Transport among Hunter-gatherers and its Implication for Plio-Pleistocene Hominid Carcasss Foraging and Site Use". Journal of Taphonomy 3 (3): I35158.

Hill, K. y J. Padwe. 2000. "The sustainability of Ache hunting in the Mbaracayu Reserve, Paraguay". En Hunting for Sustainability in Tropical Forests, editado por J. G. Robinson y E. L. Bennett, pp. 79-105. Columbia University Press, New York.

Kraglievich, L. y C. Rusconi. 1931. "Restos de vertebrados vivientes y extinguidos hallados por los señores $E$. R.Wagner y hermano en túmulos precolombinos de Santiago del Estero". Physis 10(36): 248-252.

Madrigal, T. C. 2004. "The Derivation and Application of White-Tailed Deer Utility Indices and Return Rates". Journal of Taphonomy 2: 185-199.

Madrigal, T. C. y J. Z. Holt. 2002. "White-tailed deer meat \& marrow return rates and their application to Eastern Woodlands archaeology". American Antiquity, 67(4):745-759.

Medina, M. y S. Pastor. 2012. "Zooarqueología de sitios residenciales tardíos de las sierras de Córdoba (Argentina, ca. I 100-300 AP): avances y perspectivas". En Temas de Arqueología 3: Estudios tafonómicos y zooarqueológicos (II), compilado por A. Acosta, Loponte, D. y L. Mucciolo, pp. 45-66. Instituto Nacional de Antropología y Pensamiento Latinoamericano, Buenos Aires.

Mengoni Goñalons, G. 1991. "La llama y sus productos primarios”. Arqueología. Revista de la Sección Prehistoria I:179-196

Merino, M L. y R. V. Rossi. 2010. "Origin, systematics and morphological radiation". En Neotropical Cervidology. Biology and medicine of Latin American deer, editado por J. M. B. Duarte y S. González, pp. 2-I I. FUNEP/IUCN, Brasil.

Metcalfe, D. y K. T. Jones. 1988. “A reconsideration of animal body-part utility indices". American Antiquity 53: 486-504.

Nogueira De Queiroz, A. y O. A. De Carvalho. 2008. "Problems in the interpretation of Brazilian archaeofaunas: Different contexts and the important role of taphonomy". Quaternary International 180: 75-89

Outram, A. y P. Rowley-Conwy. 1998. "Meat and Marrow Utility Indices for Horse (Equus)". Journal of Archaeological Science 25: 839-849.

Paucke, F. 1944. Hacia Allá y para Acá. Una estadía entre los indios Mocobíes, 1749-1767. Vol. III. Universidad Nacional de Tucumán, Tucumán.

Santini, M. y L. De Santis. 2012. "Investigaciones zooarqueológicas en la provincia de Chaco (Argentina)". En Temas de Arqueología 3: Estudios tafonómicos y zooarqueológicos (II), compilado por A. Acosta, Loponte, D. y L. Mucciolo, Pp. 137-165. Instituto Nacional de Antropología y Pensamiento Latinoamericano. Buenos Aires. 